\title{
Commentary
}

\section{Is Exenatide the Next Big Thing in Parkinson's Disease?}

\author{
Tanya Simuni ${ }^{\mathrm{a}, *}$ and Patrik Brundin ${ }^{\mathrm{b}, \mathrm{c}}$ \\ ${ }^{\mathrm{a}}$ Northwestern University Feinberg School of Medicine, Chicago, IL, USA \\ ${ }^{\mathrm{b}}$ Laboratory for Translational Parkinson's Disease Research, Center for Neurodegenerative Science, Van Andel \\ Research Institute, Grand Rapids, MI, USA \\ ${ }^{\mathrm{c}}$ Neuronal Survival Unit, Department of Experimental Medical Science, Wallenberg Neuroscience Center, Lund, \\ Sweden
}

\begin{abstract}
A recent study by Aviles-Olmos and colleagues suggests that 12 months of treatment with the glucagon-like peptide-1 receptor agonist exenatide improves motor and cognitive symptoms in Parkinson's disease (PD), and that the effect persists as long as 12 months after termination of the treatment. Due to the lack of a placebo control, one cannot exclude that the observed differences between patients receiving daily injections of exenatide and non-treated controls are due to a placebo effect. We discuss that large group differences in two independent functional measures remain for at least 12 months following the cessation of exenatide treatment and that this warrants a double-blind placebo-controlled trial with exenatide in PD.
\end{abstract}

While substantial progress has been made in the development of effective symptomatic therapies for Parkinson's disease (PD), no drug therapies have been proven to slow or halt the progression of symptoms. Several clinical trials have been undertaken at breathtakingly great financial cost, yet disease-modifying treatments remain the most important unmet need in PD.

Experimental research from the past decade has highlighted the possibility that exendin-4 has multiple beneficial effects in damaged or disease-stricken brains [1]. For example, exendin-4 has been suggested to interact with the glucagon-like peptide-1 (GLP-1) receptor to improve mitochondrial function, modulate neuroinflammation, increase neurogenesis, and enhance synaptic function. In this issue, Aviles-Olmos

\footnotetext{
${ }^{*}$ Correspondence to: Tanya Simuni, Northwestern University Feinberg School of Medicine, Abbott Hall, 11th Floor 1126, 710 North Lake Shore Drive, Chicago, IL 60611, USA. Tel.: +312 503 2970; Fax: +312 908 5073; E-mails: TSimuni@nmff.org; patrik.brundin@vai.org (Patrik Brundin).
}

and co-workers [2] report on a 24-month follow-up of the previously published pilot study of injections of exenatide (which contains exendin-4) as a potential disease-modifying intervention in PD [3]. The initial study was based on the rationale that exenatide, which is approved for treatment of diabetes mellitus type 2, is neuroprotective and promotes functionally beneficial neuroplasticity in animal models of neurodegeneration (including rodent models of PD) through one or more of the GLP-1 receptor mediated effects mentioned above [4]. Furthermore, exenatide has a favourable safety profile, with only relatively mild gastrointestinal side effects (including nausea and weight loss) as frequent adverse events [5].

The current study was designed as an open-label, proof-of-concept, 12-month treatment in a cohort of subjects with moderately advanced PD. Patients were randomized to either an injectable exenatide group, with exenatide given in addition to their regular PD treatment, or to a control group that received only the conventional PD treatment. Due to the high cost of 
manufacturing a placebo injection device, the control group did not receive placebo injections. The clinical outcome parameters included the Movement Disorders Society Unified Parkinson's Disease Rating Scale (MDS-UPDRS) Part III in the medications OFF state and ON state, as well as Part I-II, cognitive outcomes as measured by Mattis Dementia Rating Scale (DRS-2) and the PDQ39 quality of life scale. The MDS-UPDRS motor score was assessed on recorded video by neurologists who were blinded to the treatment assignment, and due to this procedure it was not possible to assess rigidity in a blinded fashion.

As reported previously, the study demonstrated significant and clinically meaningful differences between the scores for the exenatide-treated group and the controls for both motor and cognitive symptoms 12 months after the start of exenatide treatment. At 14 months, when the patients had been off exenatide for 2 months, the exenatide-treated and control groups still differed from each other. The authors concluded in the first report in 2013 that the study supported potential disease-modifying benefits of exenatide in PD, but they also clearly acknowledged the major limitation of the lack of a placebo arm [3]. The follow-up study, reported in the current issue, describes data obtained at 24 months after the start of the study [2]. This followup benefits from the facts that the investigators retained all originally enrolled participants and included the full scope of the outcome measures collected at 12 and 14 months. The data demonstrate persistent and statistically significant benefit in the original exenatide group versus controls in motor disability assessed by the MDS-UPDRS Part III (medications OFF state), as well as Part I-II, and in cognitive functions measured by Mattis DRS-2. In the PDQ39 scale, there were no differences between the exenatide-treated patients and the controls at 24 months, which is in full agreement with the findings at 12 and 14 months.

So what conclusions can one draw considering the lack of the placebo arm in the study design? In light of the long-term follow-up, three important points should be discussed: symptomatic effects of exenatide, observer bias, and placebo effects.

Does the new data set from 24 months support the idea of a biologic effect of exenatide and compensate for the lack of the placebo arm? Importantly, the long-term results speak strongly against a lingering "symptomatic" effect of exenatide treatment, which conceivably could have been present after the twomonth wash-out period reported in the first study. Potential observer bias was addressed by the singleblinded design (motor function assessment based on the blinded rating of videotaped MDS-UPDRS). Thus, the main debate should focus on whether there might be a lingering placebo effect in the exenatide group. Specifically, could patients given daily exenatide injections for one year display a placebo response as long as one additional year after cessation of the injections?

In the blinded rating, the size of the difference between the groups is as high as 5.6 points $(95 \% \mathrm{CI}$, $2.2-9.0 ; p=0.002$ ) was the MDS-UPRDS motor score, excluding the rigidity score (not possible to rate on the videos), in the medications OFF state. When a neurologist who knew which patients received exenatide rated rigidity, the difference between the groups at 24 months was 8.0 points in the MDS-UPDRS part III. These differences were stable and consistent between the 12- and 24-month evaluation points, which argues against a pure placebo effect. We are not aware of any published description of a placebo effect in PD that has been maintained for one year after patients were told they were not on active treatment. One should also note that while significant group differences were observed in MDS-UPDRS part III and Mattis DRS-2 scales, the PDQ39 scores never differed between the exenatidetreated and control groups. If the group differences in the motor and cognitive scales were due to placebo effects, one might have expected that there would be equally conspicuous group differences in the patient completed quality of life questionnaire (PDQ39).

Notwithstanding the promising nature of the results, it has to be emphasized that placebo effects can be highly significant and long-standing in PD. Therefore one should not jump to premature conclusions about the potential benefits of exenatide in PD. Multiple earlier clinical trials in PD have shown that placebo effects can result in $50 \%$ reductions in the motor UPDRS score and last up to 6 months from the start of the study [6]. Overall, placebo responses are more pronounced with invasive procedures and in PD patients with advanced disease [7]. Placebo can induce biochemical changes in the dopamine system, as documented by positron emission tomography and single-cell recordings in the subthalamic nucleus neurons [8,9]. Those observations, however, have been made in acute paradigms lasting minutes to hours, not months or years. Taken together, while placebo effects ought to have diminished 12 months after drug withdrawal so that the exenatide-treated and control groups no longer differed, a lingering placebo effect cannot be excluded. In addition, there is a novel important observation of the "lessebo effect", which is a negative expectation related to receiving a placebo or no therapy, that could have impacted the control group [10]. 
A Phase II placebo-controlled study on exenatide in PD is to be launched shortly (Clintrials.gov NCT01971242). The current data strengthens the rationale for such a study and was likely important when securing funding for that new Phase II placebocontrolled trial. In conclusion, the present study by Aviles-Olmos could represent a milestone if future controlled trials provide evidence supporting a diseasemodifying effect of exenatide and lead to a revolution in PD therapy. Furthermore, views on the value of small pilot trials with extended washout periods might shift, making them more generally accepted as an alternative when screening potential disease-modifying agents for use against progressive neurodegenerative diseases.

\section{DISCLOSURES}

TS has received research funding from Michael J. Fox Foundation for Parkinson's Research, National Institutes of Health, National Parkinson Foundation, and consulting honoraria from National Parkinson Foundation, Teva Pharmaceuticals, Pfizer, Harbor, UCB, IMPAX, Eli Lilly and Company, Allergan, Merz Inc, and US Worldmeds.

$\mathrm{PB}$ is a paid consultant for $\mathrm{H}$ Lundbeck $\mathrm{A} / \mathrm{S}$, TEVA Pharmaceuticals and Renovo Neural Inc. He is a cofounder of the biotechnology companies ParkCell $\mathrm{AB}$ and Acousort AB. His research team has funded collaborations with Metabolic Development Solutions Company, BioArctic Neuroscience AB, Roche and TEVA Pharmaceuticals.

\section{REFERENCES}

[1] Mattson MP (2014) Interventions that improve body and brain bioenergetics for Parkinson's disease risk reduction and therapy. J Parkinsons Dis, 4, 1-13.
[2] Aviles-Olmos I, Dickson J, Kefalopoulou Z, Djamshidian A, Kahan J, Fmedsci PE, Whitton P, Wyse R, Isaacs T, Lees A, Limousin P, \& Foltynie T (2014) Motor and cognitive advantages persist 12 months after exenatide exposure in Parkinson's disease. J Parkinsons Dis. doi: 10.3233/JPD140364

[3] Aviles-Olmos I, Dickson J, Kefalopoulou Z, Djamshidian A, Ell P, Soderlund T, Whitton P, Wyse R, Isaacs T, Lees A, Limousin P, \& Foltynie T (2013) Exenatide and the treatment of patients with Parkinson's disease. J Clin Invest, 123, 27302736.

[4] Aviles-Olmos I, Limousin P, Lees A, \& Foltynie T (2013) Parkinson's disease, insulin resistance and novel agents of neuroprotection. Brain, 136, 374-384.

[5] Foltynie T, \& Aviles-Olmos I (2014) Exenatide as a potential treatment for patients with Parkinson's disease: First steps into the clinic. Alzheimers Dement, 10, S38-S46.

[6] Goetz CG, Wuu J, McDermott MP, Adler CH, Fahn S, Freed CR, Hauser RA, Olanow WC, Shoulson I, Tandon PK, Parkinson Study G, \& Leurgans S (2008) Placebo response in Parkinson's disease: Comparisons among 11 trials covering medical and surgical interventions. Mov Disord, 23, 690-699.

[7] Diederich NJ, \& Goetz CG (2008) The placebo treatments in neurosciences: New insights from clinical and neuroimaging studies. Neurology, 71, 677-684.

[8] Benedetti F, Colloca L, Torre E, Lanotte M, Melcarne A, Pesare M, Bergamasco B, \& Lopiano L (2004) Placeboresponsive Parkinson patients show decreased activity in single neurons of subthalamic nucleus. Nat Neurosci, 7, 587588.

[9] de la Fuente-Fernandez R, Ruth TJ, Sossi V, Schulzer M, Calne DB, \& Stoessl AJ (2001) Expectation and dopamine release: Mechanism of the placebo effect in Parkinson's disease. Science, 293, 1164-1166.

[10] Mestre TA, Shah P, Marras C, Tomlinson G, \& Lang AE (2014) Another face of placebo: The lessebo effect in Parkinson disease: Meta-analyses. Neurology. doi: 10.1212/ WNL.0000000000000340 